\title{
Non-resonant terahertz field enhancement in periodically arranged nanoslits
}

Novitsky, Andrey; Ivinskaya, Aliaksandra; Zalkovskij, Maksim; Malureanu, Radu; Jepsen, Peter Uhd; Lavrinenko, Andrei

Published in:

Journal of Applied Physics

Link to article, DOI:

$10.1063 / 1.4757024$

Publication date:

2012

Document Version

Publisher's PDF, also known as Version of record

Link back to DTU Orbit

Citation (APA):

Novitsky, A., Ivinskaya, A., Zalkovskij, M., Malureanu, R., Jepsen, P. U., \& Lavrinenko, A. (2012). Non-resonant terahertz field enhancement in periodically arranged nanoslits. Journal of Applied Physics, 112, 074318.

https://doi.org/10.1063/1.4757024

\section{General rights}

Copyright and moral rights for the publications made accessible in the public portal are retained by the authors and/or other copyright owners and it is a condition of accessing publications that users recognise and abide by the legal requirements associated with these rights.

- Users may download and print one copy of any publication from the public portal for the purpose of private study or research.

- You may not further distribute the material or use it for any profit-making activity or commercial gain

- You may freely distribute the URL identifying the publication in the public portal

If you believe that this document breaches copyright please contact us providing details, and we will remove access to the work immediately and investigate your claim. 


\section{AIP Appilied Physics}

\section{Non-resonant terahertz field enhancement in periodically arranged nanoslits}

Andrey Novitsky, Aliaksandra M. Ivinskaya, Maksim Zalkovskij, Radu Malureanu, Peter Uhd Jepsen et al.

Citation: J. Appl. Phys. 112, 074318 (2012); doi: 10.1063/1.4757024

View online: http://dx.doi.org/10.1063/1.4757024

View Table of Contents: http://jap.aip.org/resource/1/JAPIAU/v112/i7

Published by the American Institute of Physics.

\section{Related Articles}

Ultra-low power electrically reconfigurable magnetoelectric microwave devices

J. Appl. Phys. 112, 073915 (2012)

Broadband and ultrathin screen with magnetic substrate for microwave reflectivity reduction Appl. Phys. Lett. 101, 154101 (2012)

Tunable, narrow-band, all-metallic microwave absorber

Appl. Phys. Lett. 101, 141115 (2012)

Insertable system for fast turnaround time microwave experiments in a dilution refrigerator

Rev. Sci. Instrum. 83, 093904 (2012)

Coherent terahertz radiation from high-harmonic component of modulated free-electron beam in a tapered twoasymmetric grating structure

Appl. Phys. Lett. 101, 123503 (2012)

\section{Additional information on J. Appl. Phys.}

Journal Homepage: http://jap.aip.org/

Journal Information: http://jap.aip.org/about/about_the_journal

Top downloads: http://jap.aip.org/features/most_downloaded

Information for Authors: http://jap.aip.org/authors

\section{ADVERTISEMENT}

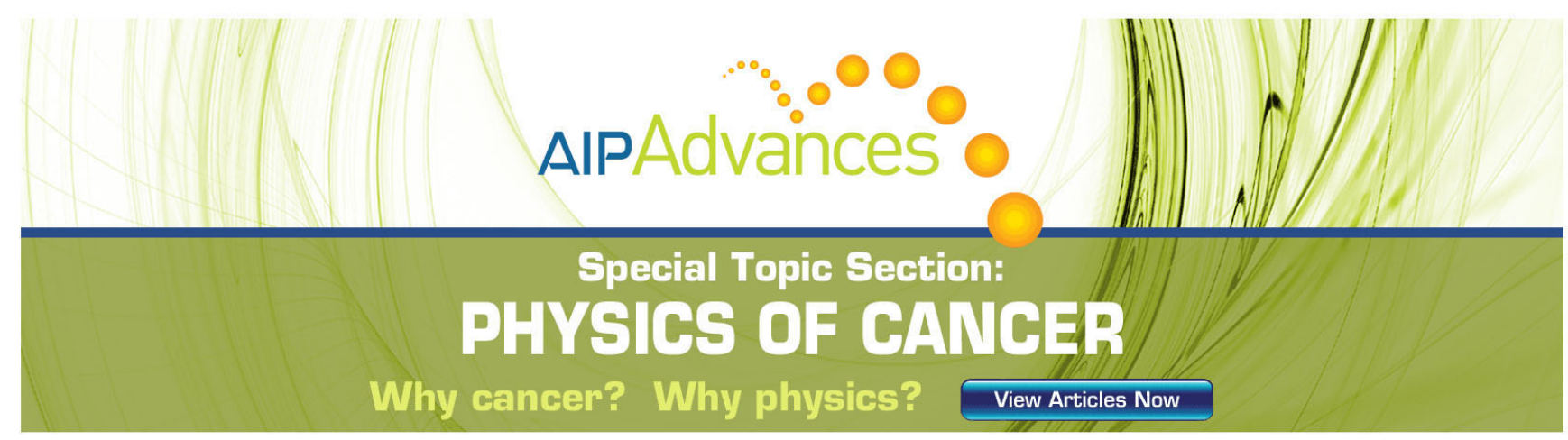




\title{
Non-resonant terahertz field enhancement in periodically arranged nanoslits
}

\author{
Andrey Novitsky, ${ }^{a)}$ Aliaksandra M. Ivinskaya, Maksim Zalkovskij, Radu Malureanu, \\ Peter Und Jepsen, and Andrei V. Lavrinenko \\ DTU Fotonik, Department of Photonics Engineering, Technical University of Denmark, Ørsteds Plads 343 , \\ DK-2800 Kgs. Lyngby, Denmark
}

(Received 4 January 2012; accepted 5 September 2012; published online 8 October 2012)

\begin{abstract}
We analyze ultra strong non-resonant field enhancement of $\mathrm{THz}$ field in periodic arrays of nanoslits cut in ultrathin metal films. The main feature of our approach is that the slit size and metal film thickness are several orders of magnitude smaller than the wavelength $\lambda$ of the impinging radiation. Two regimes of operation are found. First, when the grating period $P \ll \lambda$, frequency-independent enhancement is observed, accompanied by a very high transmission approaching unity. With high accuracy, this enhancement equals the ratio of $P$ to the slit width $w$. Second, when the grating period approaches the $\mathrm{THz}$ wavelength but before entering the Raleigh-Wood anomaly, the field enhancement in nanoslit stays close to that in a single isolated slit, i.e., the well-known inversefrequency dependence. Both regimes are non-resonant and thus extremely broadband for $P<\lambda$. The results are obtained by the microscopic Drude-Lorentz model taking into account retardation processes in the metal film and validated by the finite difference frequency domain method. We expect sensor and modulation applications of the predicted giant broadband field enhancement. (C) 2012 American Institute of Physics. [http://dx.doi.org/10.1063/1.4757024]
\end{abstract}

\section{INTRODUCTION}

Investigation of the interaction between light and slits cut in metals has a long history. Starting from the works of Wood and Rayleigh, ${ }^{1,2}$ it extended through the theory of metal apertures by Bethe ${ }^{3}$ and its improvement ${ }^{4-6}$ to modern nanophotonics problems. The interest in the optics of metal slits was greatly renewed in 1998 with the observation of extraordinary optical transmission (EOT). ${ }^{7,8}$ Two mechanisms for the resonance peaks to appear in the transmission spectra of metal apertures were found in Ref. 9: coupled surface plasmon-polaritons on both surfaces of the metal film and cavity-like modes appearing when resonance condition is satisfied for the height ${ }^{10-12}$ or width ${ }^{13}$ of a slit. One of the important aspects of these resonance effects is a strong light concentration in the air slits; for example, field enhancement of up to 60 was achieved in specially designed charge and current reservoirs in visible. ${ }^{14}$ Recent publications have reported on high field enhancement in metallic nanoslits, when they are illuminated by a $\mathrm{THz}$ electromagnetic wave. ${ }^{15-19}$ Park et al. ${ }^{19}$ observed a 170 -fold field enhancement through an array of slits studied in terms of broadband filtering; an enhancement of about 430 was expected in the near field of periodic rectangular slits. ${ }^{18}$

At $\mathrm{THz}$ frequencies, bulk $20-100 \mathrm{~nm}$ thick metal films become non-transparent and reflects most of the incident radiation (in the visible $20 \mathrm{~nm}$, film can transmit up to 50\%). The ability of metal to effectively transmit long-wavelength radiation by a nanometer-thick film with low absorption is of good use when we are interested in strong field concentration in air gaps in the metal.

Surprisingly, high field enhancements of about $10^{3}$ and higher are accumulated inside isolated slits in the non-resonant regime at low frequencies. ${ }^{17,20}$ The enhancement has an inverse frequency dependence confirmed by the quasistatic model of a local capacitor. ${ }^{17}$ The longer the wavelength, the larger part of the metal is involved in gathering of electrons near the slit and thus in the field concentration process. In our study, non-resonant field enhancement in arrays of slits is investigated. Height and period of the slits and the slit width are chosen smaller than $\lambda$ to not step into the resonance regime.

The paper consists of analytical and simulation parts. After a brief phenomenological analysis of the problem in Sec. II, a microscopic analytical model dealing with the dynamics of an individual electron in a metal is built up in Sec. III. The structures are further investigated and the model is validated numerically with the finite-difference frequencydomain (FDFD) method (Sec. IV). We find the electric field enhancement and transmission both for periodically arranged slits and for a single slit. Section V concludes the paper.

\section{SIMPLE MODEL OF FREQUENCY-INDEPENDENT FIELD ENHANCEMENT}

We consider a metal film of thickness $h$ with a periodic array (period $P$ ) of slits of width $w$ in the $x$ direction and infinite extent in the $y$ direction. The structure is illuminated by a monochromatic plane wave of frequency $f$ polarized perpendicular to the slits, in the $x$ direction (Fig. 1). In the numerical simulations, we define the field enhancement $G$ as the amplitude of electric field averaged over the central line of the slit normalized to the amplitude $E^{i n c}$ of the incident radiation

$$
G=\frac{\left.\langle E\rangle_{\text {slit }}\right|_{z=0}}{E^{\text {inc }}} \approx \frac{\left.\int_{w} E_{x}\right|_{z=0} d x}{w E^{\text {inc }}} .
$$




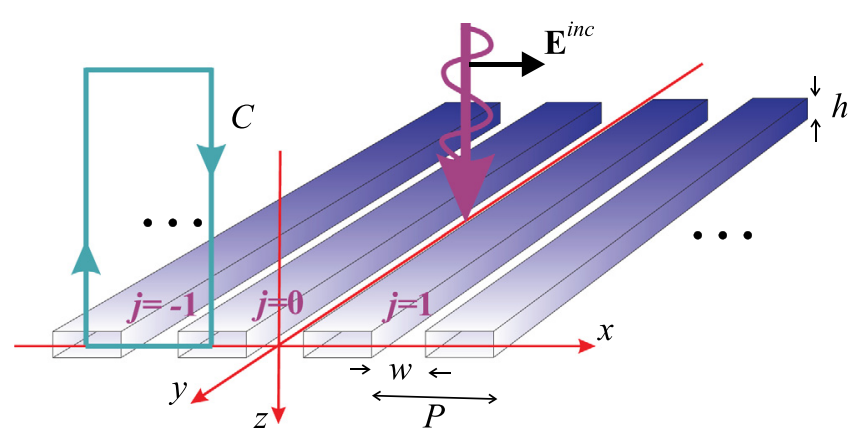

FIG. 1. Sketch of the periodic slit array illuminated by $p$-polarized light. Slits are infinitely long in the $y$-direction. Contour $C$ is intended for the enhancement estimation Eq. (2) in the static regime.

In the low frequency limit, field enhancement $G$ can be estimated by considering the plane wave field as a quasistatic. The electrostatic field is a potential field and, therefore, work $A$ done by this field along any closed contour must be zero. We choose rectangular contour $C$ passing symmetrically through one period of the perforated metal and closing at infinity (Fig. 1). Since there is no field in metal and the work along the $z$-sides of $C$ can be neglected, the full work $A_{C}$ over contour $C$ can be represented as the sum of the works in the slit $-\left\langle E_{x}\right\rangle w$ and over one period distance at infinity $E^{i n c} P$. Equation $A_{C}=0$ gives us field enhancement in terms of the period of the array $P$ and slit width $w$ as

$$
G=\left\langle E_{x}\right\rangle / E^{i n c}=P / w .
$$

Therefore, the enhancement in periodic structures $G=P / w$ depends on the period of grating and size of the opening while slab thickness does not play any role. In the static approximation, a uniform electric field on both sides of the perforated metal at infinity implies also possibility of enhanced transmission, what is in agreement with the transmission increase in Fig. 17(a) from Ref. 8 at the $f \rightarrow 0$ limit.

The realization of the quasistatic enhancement is illustrated in Fig. 2, where all quantities are normalized to the same characteristics of the incident plane wave. The electric field does not penetrate into the metal (the metal can be treated as a perfect electric conductor), and $E_{x}$ component (Fig. 2(a)) is strongly enhanced inside the slit with maximum relative amplitude reaching 200 near the sharp edges of the slit. Another component $E_{z}$ (Fig. 2(b)) is strongly concentrated in the vicinity of metal corners and has antisymmetric field distribution around the node $x=0, z=0$. Thus, $E_{z}$ is vanishingly small along the $z=0$ line, which justifies our definition of $G$. Being close to unity, the magnetic field does not participate significantly in the scattering by the grating (Fig. 2(c)), and the Poynting vector components are proportional to the electric field as $S_{x} \sim E_{z}$ and $S_{z} \sim E_{x}$. Inside the slit, $S_{z}$ component dominates in the total energy flux density and $|\mathbf{S}|$ is similar to $E_{x}$ distribution. Thus, the electric field and the Poynting vector in the grating have similar enhancements in contrast to single slit case, ${ }^{20}$ where flux enhancement is less than the electric field enhancement.

To know the distribution of $E_{x}$ along the middle line of the aperture, Fig. 2(e) compares three different slit widths providing the same enhancement of 100 calculated via Eq. (1). Only in the case of an extremely narrow 10-nm-wide slit, we see the uniform field, while opening of the aperture leads to a drop of the field amplitude well below the average value in the central region.

The calculations for Fig. 2 were made with the 2D FDFD method with perfectly matched layers and squeezetransformed layers as absorbing boundary conditions in the $z$-directions. ${ }^{21}$ In Fig. 2(f), we see the discretization mesh; grid steps near the slit are around $0.1-0.4 \mathrm{~nm}$ and are gradually stretched to reach 20 times smaller mesh compared to the rest of the structure.

\section{MICROSCOPIC MODEL}

\section{A. Field enhancement}

To build up an analytical model of the $\mathrm{THz}$ field enhancement in the nano-sized slits, we employ the DrudeLorentz-based theory of electrons in metal. An electron in the metal film illuminated by a plane wave is affected by several forces: driving force $e E^{(i n c)}$, friction force $-m \gamma \dot{\xi}$, and screening force $F$ as a result of pushing back electrons by the electrical forces of the displaced charges, ${ }^{22,23}$ where $\xi$ is the electron displacement, "dot" stands for the time differentiation, $\gamma$ is the collision frequency, $e$ and $m$ are electron's charge and mass, respectively. Then the equation of motion takes the form

$$
m \ddot{\xi}+m \gamma \dot{\xi}=e E^{(i n c)}+F .
$$

The charges accumulated at the slit edges give rise to the screening force $F$. Assuming homogeneous charging of the slit edges the electric field of an edge can be approximated by that of a $y$-infinite strip of width $h$ (Fig. 1). In Appendix A, we derive the dominant component $E_{x}$ of electric field of such a strip positioned at $x=x_{0}$. In the periodic array of slits, we have infinite number of positively charged strips (at $\left.x_{+}^{(j)}=x+w / 2-j P, j=-\infty, \infty\right)$ and infinite number of negatively charged strips (at $x_{-}^{(j)}=x-w / 2-j P, j=-\infty$, $\infty$ ). For the slit $j=0$ (see Fig. 1), the total electric field is the sum of the contributions from all slits edges (in this paper we use Gaussian units):

$$
E_{x}(x, z)=2 \eta f(x, z)
$$

where surface charge density can be estimated as $\eta=e N \xi$, $N$ is the volume electron density of the metal. In function,

$$
\begin{aligned}
f(x, z)= & \sum_{j=-\infty}^{\infty}\left[\arctan \left(\frac{z_{+}}{x_{+}^{(j)}}\right)+\arctan \left(\frac{z_{-}}{x_{+}^{(j)}}\right)\right. \\
& \left.-\arctan \left(\frac{z_{+}}{x_{-}^{(j)}}\right)-\arctan \left(\frac{z_{-}}{x_{-}^{(j)}}\right)\right],
\end{aligned}
$$

$z_{ \pm}=h / 2 \pm z$ and positive terms correspond to the positively charged edges (surface density $+\eta$ ), while negative terms stand for the negatively charged edges $(-\eta)$.

We define the screening force as the sum of the forces from all the slit edges averaged over the metal volume 

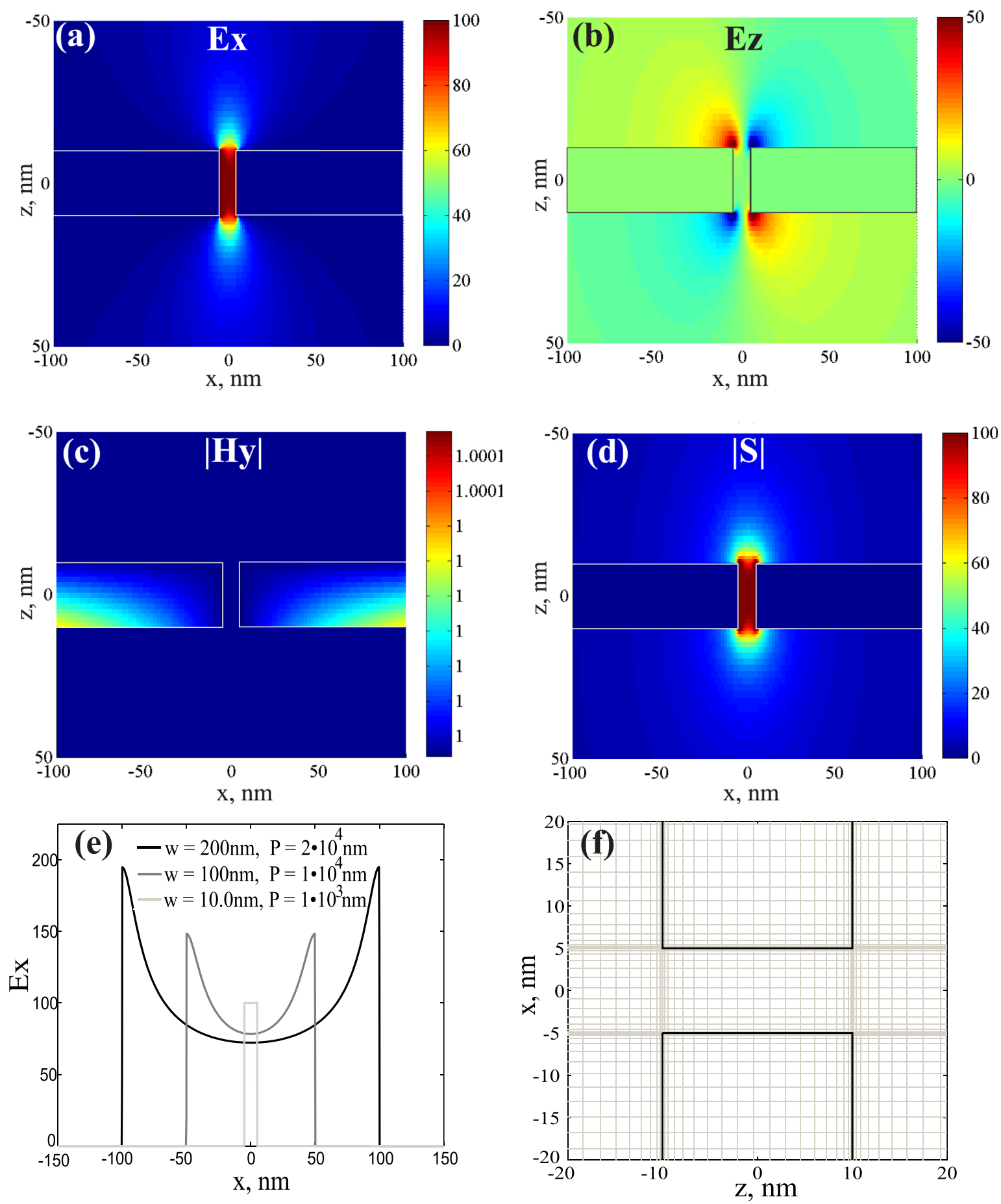

FIG. 2. FDFD simulations of components (a) $E_{x}$ and (b) $E_{z}$ of the electric field, absolute values of (c) the magnetic field $H_{y}$ and (d) the Poynting vector $\mathbf{S}=$ $\operatorname{Re}\left(\mathbf{E} \times \mathbf{H}^{*}\right)$ near the 10-nm-wide slit of thickness $h=50 \mathrm{~nm}$ in a gold grating with a period of $1 \mu \mathrm{m}$ at a frequency $f=0.01 \mathrm{THz}$ resulting in $G=100$. Bounds for color maps are chosen so to show better peculiarities of field distribution. (e) Profiles of the electric field component $E_{x}$ in different slits, all giving enhancement of 100. Parameters of gratings are given in the legend. (f) Mesh refinement (thin, grey lines) at the metal-slit boundaries (thick, black lines).

$$
F=\frac{1}{(P-w) h} \int_{-P+w / 2}^{-w / 2} \int_{-h / 2}^{h / 2} e E_{x}^{r e t}(x, z) d x d z
$$

The superscript "ret" denotes that we take into account the retardation effects, which are introduced as the imaginary part of the force

$$
F=\frac{2 e \eta}{(P-w) h} \int_{-P+w / 2}^{-w / 2} \int_{-h / 2}^{h / 2}\left(1-\mathrm{i} \omega \frac{R}{c}\right) f(x, z) d x d z
$$

where factor $1-\mathrm{i} \omega R / c$ is the first Taylor expansion terms of the exponential retardation $\exp (-\mathrm{i} \omega R / c)$. Characteristic distance $R$ is connected with the period of the slit array $P$ and manages the enhancement regime. The coefficient of proportionality between $R$ and $P$ can be obtained from the numerical simulation.

Harmonic incident wave $E^{(i n c)} \sim \mathrm{e}^{-\mathrm{i} \omega t}$ requires harmonic electron displacement $\xi \sim \mathrm{e}^{-\mathrm{i} \omega t}$. Therefore, $-\mathrm{i} \omega \xi$ is identical with $\dot{\xi}$, and the force takes the form 


$$
F=-m \omega_{0}^{2} \xi-m \gamma_{0} \dot{\xi}
$$

where

$$
\begin{aligned}
& \omega_{0}^{2}=-\frac{\omega_{p}^{2}}{2 \pi(P-w) h} \int_{-P+w / 2}^{-w / 2} \int_{-h / 2}^{h / 2} f(x, z) d x d z, \\
& \gamma_{0}=\frac{R}{c} \omega_{0}^{2} .
\end{aligned}
$$

Plasma frequency is defined as $\omega_{p}=\sqrt{4 \pi N e^{2} / m}$. The screening force $F$ (Eq. (8)) consists of an oscillatory term and a friction term. The oscillatory term describes collective oscillations of the electrons with frequency $\omega_{0}$. The oscillation frequency is large for small periods $P$. When the period $P$ gets larger, the integral in Eq. (9) does not change much, and $\omega_{0}^{2}$ behaves like $1 / P$. The friction term results from the retardation effects. For large periods, e.g., for a single slit, $R \sim P$ and $\omega_{0}^{2} \sim 1 / P$, so that the dependence on period $P$ disappears ${ }^{24}$ in the damping rate $\gamma_{0}$.

With the screening force (8), the equation of motion (3) for the electron reads

$$
\ddot{\xi}+\tilde{\gamma} \dot{\xi}+\omega_{0}^{2} \xi=(e / m) E^{(i n c)},
$$

where the effective decay rate $\tilde{\gamma}=\gamma+\gamma_{0}$ is introduced. Remembering that the driving field and electron displacement are harmonic in time $\left(\sim \mathrm{e}^{-\mathrm{i} \omega t}\right)$, solution of Eq. (10) takes the form

$$
\xi=\frac{e / m}{-\omega^{2}-\mathrm{i} \tilde{\gamma} \omega+\omega_{0}^{2}} E^{(i n c)} .
$$

It is worth to notice that frequency $\omega_{0}$ describes a sort of plasmonic oscillation. It is defined by the plasma frequency $\omega_{p}$ that is much bigger than the frequency of the incident $\mathrm{THz}$ field. Therefore, the oscillations are off-resonant. The decay frequency $\gamma_{0}$ is proportional to the square of oscillation frequency and, therefore, is extremely large, too. Thus, $\gamma_{0} \gg \gamma$ and the approximation $\tilde{\gamma} \approx \gamma_{0}$ holds well. The plasma frequency enters both in the numerator and denominator of the surface charge density $\eta=e N \xi$ (and the field created by this charge). That is why, the field in the slit is almost insensitive to the metal parameters $\gamma$ and $\omega_{p}$, and the metal with a very good accuracy can be regarded as a perfect electric conductor.

The field enhancement $G$ is defined as the ratio of the field amplitude averaged over the slit to the incident field

$$
G=\left|\frac{E^{(i n c)}+\left\langle E_{x}\right\rangle_{s l}}{E^{(i n c)}}\right|=\left|1+\frac{1}{w h} \int_{-w / 2}^{w / 2} \int_{-h / 2}^{h / 2} \frac{E_{x}(x, z)}{E^{(i n c)}} d x d z\right| .
$$

Though this definition differs from that used in numerical simulations (see Eq. (1)), the results of both analytical and numerical calculations can be compared in the case of narrow slits. Remember that $E_{x}$ here is the electric field of the charged slit edges. To get simple closed-form expressions, we will use the field at the center of the slit $(x=0, z=0)$ instead of the averaged field,

$$
\begin{aligned}
G_{c}= & \mid 1+\frac{2 \omega_{p}^{2} / \pi}{-\omega^{2}-\mathrm{i} \tilde{\gamma} \omega+\omega_{0}^{2}}\left(\arctan \left(\frac{h}{w}\right)\right. \\
& \left.+\sum_{j=1}^{\infty}\left[\arctan \left(\frac{h / 2}{\tilde{x}_{+}^{(j)}}\right)-\arctan \left(\frac{h / 2}{\tilde{x}_{-}^{(j)}}\right)\right]\right) \mid,
\end{aligned}
$$

where $\tilde{x}_{ \pm}^{(j)}= \pm w / 2+j P$.

For large enough periods $P \gg w, h$, frequency $\omega_{0}$ can be derived as it is done in Appendix B. Then in the quasistatic limit $\omega_{0} \gg \sqrt{\tilde{\gamma} \omega} \gg \omega$, one obtains Eq. (2) discussed in Sec. II, $G_{c}=P / w$.

For a single slit, the period tends to infinity and, therefore, the oscillation frequency $\omega_{0}$ is negligible. The friction term $m \tilde{\gamma} \dot{\xi}$ dominates resulting in

$$
G_{c}=\frac{2 \omega_{p}^{2}}{\pi \tilde{\gamma} \omega} \arctan \left(\frac{h}{w}\right)
$$

It should be noted that we have omitted the sum in Eq. (13) for infinitely large periods $P$. The inverse frequency dependence for a single slit well known from the literature $15,16,20,24$ is clearly noticed. The geometric parameters $w$ and $h$ enter not only to the explicitly written arctangent function but also to the quantity $\tilde{\gamma}=\gamma+\gamma_{0}$ (remember that $\gamma_{0}$ is defined by Eq. (9)).

Characterization of the periodic grating along the elaborated model is presented in Fig. 3 .

The constant values of $G$ calculated at lower frequencies depend on the oscillation frequency $\omega_{0}$, while the decaying shape of the curves in Fig. 3(a) is defined by the retardation distance $R$, which is taken as $R=2 P$ to be consistent with numerical simulations (Figs. 5 and 6).

According to our microscopic model, the enhancement factor exhibits linear dependence on the period $P$ (see Fig. 3(b)). The model allows us to take into consideration any number of slits by including required number of terms in the series (see Eq. (5)). The integer number $M$ indicates, how many slits we want to include. When $M=0$, we take only one slit $j=0$ (see Fig. 1). When $M=1$, one slit on the right $j=+1$ and one slit on the left $j=-1$ are added, and so on. The results converge extremely fast. From Fig. 3(b), we conclude that it is sufficient to include the nearest slits only, because the two more slits $(M=2)$ result in approximately the same curve as in the case $M=1$. The enhancement factor $G_{c}$ defined by field at the slit center does not strongly differ from the enhancement with field averaging across the slit (Eq. (12)). Therefore, the use of $G_{c}$ for closed-form estimations is justified. When the period $P$ becomes very large, the linear dependence turns into a constant value (see the inset in Fig. 3(b)). The saturation behavior is expected, as there is no $P$-dependence for a single slit. Exact inverse-width dependencies of the $G$-factor on the slit width are demonstrated in Fig. 3(c). Period $P=10 \mathrm{~mm}$ approximates the case of the single slit and corresponds to the largest enhancement. When the slit width is equal to the period $P$ (no metal), the field enhancement approaches unity. As one can see in Fig. 3(d), there is almost no dependence on the metal thickness $h$, as we have already heuristically pointed in Sec. II. The ranges of the model are approximately between $10 \mathrm{~nm}$ and 

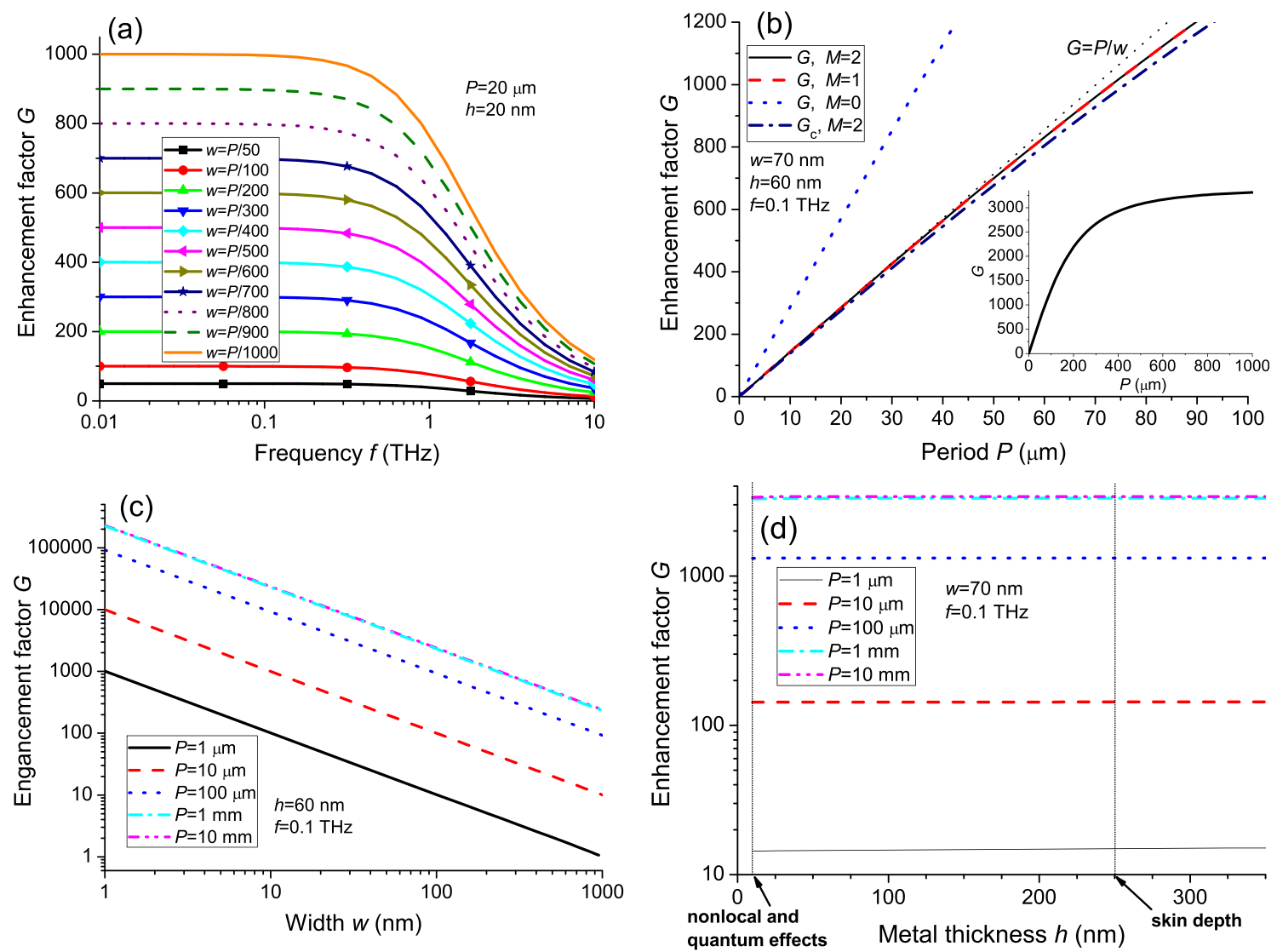

FIG. 3. (a) Enhancement factor $G$ (see Eq. (12)) for different slit widths (compare with numerical simulation in Fig. 6(a)). (b) Enhancement factor for slitaverage field $G$ and field at the slit center $G_{c}$, if $2 M$ slits act on the slit under consideration. In the inset, the long periods $P$ approaching to the wavelength are considered. Enhancement factor versus (c) slit width and (d) metal thickness.

the skin depth of the metal. For thinner metal films, the nonlocal and quantum effects start to play important roles. Thicker metal slabs bring us to the perfect conductor model of the metal (Fig. 3(d)).

The apparent absence of a thickness dependence is rather unusual, because conventional diffraction theory predicts the dependence. Let us consider the same situation $w<$ $h \ll \lambda=2 \pi c / \omega$ (metallic capacitor) in two cases, perfect electric conductor as a metal (diffraction theory) and subskin-depth Drude metal (theory of this paper, skin depth is about $250 \mathrm{~nm}$ at $0.1 \mathrm{THz}$ ).

In the approximation of the perfect conductor, the skin depth is infinitely small and the electric current density excited by the incident plane wave flows along the metallic surface, $\mathbf{j}=J_{0} \mathbf{e}_{x}(\delta(z-h / 2)+\delta(z+h / 2))$, where $J_{0}$ is constant proportional to the magnetic field at the metallic interface, $\delta(z)$ is the Dirac delta function, $\mathbf{e}_{x}$ is the unit vector along the $x$ axis. Then the conservation of the electric charge for monochromatic excitation results in the charge at the slit edge

$$
q=-\frac{c}{i \omega} \int\left(-\frac{i \omega}{c} \rho\right) d V=-\frac{c}{i \omega} \int \nabla \mathbf{j} d V=-\frac{c}{i \omega} \oint \mathbf{j} \mathbf{n} d s .
$$

The normal vector $\mathbf{n}$ is orthogonal to the interface everywhere except the slit edges $\left(\mathbf{n}=\mathbf{e}_{x}\right)$, hence we get

$$
q=-\frac{c J_{0} b}{i \omega} \int_{-h / 2}^{h / 2}(\delta(z-h / 2)+\delta(z+h / 2)) d z=-\frac{2 c J_{0} b}{i \omega},
$$

where $b$ is the slit dimension along the $y$ axis. Dividing by the area of the slit edge $b h$ determines the surface density $\sigma_{\mathrm{PEC}}$ $=2 i c J_{0} /(\omega h)$, which gives us the electric field in the slit as

$$
E_{\mathrm{PEC}}=4 \pi \sigma_{\mathrm{PEC}}=\frac{8 \pi i c J_{0}}{\omega h} .
$$

In this simple model, we indeed observe the inverse thickness dependence in accordance with Refs. 17 and 18.

In the sub-skin-depth regime of the Drude model of the metal, the electric current is approximately homogeneously distributed (because the electric field in the metal is homogeneous ${ }^{24}$ ) and is equal to $\mathbf{j}=j_{0} \mathbf{e}_{x}$. Therefore, the charge collected at the edge of the slit takes the form

$$
q=-\frac{c}{i \omega} \int j_{0} d s=-\frac{2 c j_{0} b h}{i \omega},
$$

while the surface charge density equals $\sigma_{\mathrm{D}}=2 i c j_{0} / \omega$. Then the electric field in the slit

$$
E_{\mathrm{D}}=4 \pi \sigma_{\mathrm{D}}=\frac{4 \pi i c j_{0}}{\omega}
$$

does not depend on the thickness. Thus, the thickness dependence is a consequence of the applied metal model.

\section{B. Transmission through an array of slits}

It is interesting to look at transmission of the perforated metal film, because transmission is the characteristic which 
can be directly calculated numerically or measured in experiments. To calculate transmission through the array of slits, we homogenize the metal film with cuts. Knowing the effective parameters transmission through the film with an array of slits will be easily obtained. First, we define the unit cell as a parallelepiped with dimensions $-P$ to 0 in the $x$ direction, $-P / 2$ to $P / 2$ in the $y$ direction, and $-h / 2$ to $h / 2$ in the $z$ direction, with volume $V_{\text {cell }}=P^{2} h$.

It is evident that the homogenized material is anisotropic due to different responses for $x$-polarized and $y$-polarized incident waves. Since we are interested in the transmission of an $x$-polarized incident wave, the effective parameters will be derived specifically for this situation. The electric field in the slit is the sum of the incident field and the screening field of the charged metal edges averaged over the slit

$$
E^{(s l)}=E^{(i n c)}+\left\langle E_{x}\right\rangle_{s l}=G E^{(i n c)},
$$

while the field inside the metal is the sum of the incident field and the field of the charged edges averaged over the metal

$$
E^{(m)}=E^{(i n c)}+\left\langle E_{x}\right\rangle_{m e t} .
$$

Then the electric field averaged over the unit cell is

$$
E=p_{1} E^{(s l)}+p_{2} E^{(m)},
$$

where $p_{1}=w / P$ and $p_{2}=1-w / P$ are the filling factors of air and metal, respectively.

The average $x$-component of the displacement vector in the metal film can be written in the form

$$
D=p_{1} E^{(s l)}+p_{2} \varepsilon_{m} E^{(m)},
$$

where $\varepsilon_{m}$ is the dielectric permittivity of the metal. Both in the model and simulation, we consider aperture gold films. Permittivity of gold can be modeled as

$$
\epsilon_{m}(\omega)=\epsilon_{\infty}-\frac{\omega_{p}^{2}}{\omega(\omega+i \gamma)},
$$

with $\epsilon_{\infty}=1$, plasma frequency $\omega_{p}=1.37 \times 10^{16} \mathrm{rad} / \mathrm{s}$, and collision frequency $\gamma=40.7 \times 10^{12} \mathrm{~s}^{-1}$ (see Ref. 20). We do not consider extremely thin metal films, for which the Drude model is violated and advanced hydrodynamic model should be applied. ${ }^{25-27}$ It should be noted that the field enhancement and transmission weakly depend on the Drude parameters of the metal; that is, perfect conductor model can be applied.

Electric field in the slit can be expressed in terms of the enhancement as $E^{(s l)}=G E^{(i n c)}$. $\omega_{0}$ is introduced through the averaging over the metal (see Eq. (9)), therefore, electric field averaged over the metal takes the form $\left\langle E_{x}\right\rangle_{\text {met }}=2 \eta$ $\langle f(x, z)\rangle_{\text {met }}=2 \eta\left(-2 \pi \omega_{0}^{2} / \omega_{p}^{2}\right)=-m \omega_{0}^{2} \xi$, where we use the definitions of $\eta$ and $\omega_{p}$. Then expression (19) gives $E^{(m)}$ $=E^{(i n c)}-m \omega_{0}^{2} \xi / e=\left[1-\omega_{0}^{2}(\varepsilon-1) / \omega_{p}^{2}\right] E^{(i n c)}$, where we introduce the effective metal permittivity

$$
\varepsilon(\omega)=1-\frac{\omega_{p}^{2}}{\omega^{2}+\mathrm{i} \tilde{\gamma} \omega-\omega_{0}^{2}} .
$$

By substituting $E^{(s l)}$ and $E^{(m)}$ into Eq. (21), we arrive at the dielectric permittivity of the unit cell

$$
\varepsilon_{\text {cell }}=D / E=1+4 \pi \kappa,
$$

where

$$
\kappa=\frac{1}{4 \pi} \frac{p_{2}\left(\varepsilon_{m}-1\right)\left[1-\omega_{0}^{2}(\varepsilon-1) / \omega_{p}^{2}\right]}{p_{1} G+p_{2}\left[1-\omega_{0}^{2}(\varepsilon-1) / \omega_{p}^{2}\right]} .
$$

In the homogenization procedure, the coupling of slits can be taken into account using the so-called interaction matrix. ${ }^{28}$ The interaction matrix $\hat{C}$ is introduced in terms of the relation $\mathbf{E}=\overline{\mathbf{E}}+4 \pi \hat{C} \mathbf{P}_{0}$, where $\overline{\mathbf{E}}$ is the electric field averaged over the whole periodic structure, $\mathbf{E}$ is the field averaged over the unit cell ( $E$ is its component in the $x$ direction), and $\mathbf{P}_{0}=N_{0} \mathbf{p}$ is the polarization of the medium $\left(N_{0}=1 / V_{\text {cell }}\right.$ is the number of inclusions per unit volume). The interaction matrix results from the collective action of the dipole moments $\mathbf{p}$ placed in the centers of their unit cells, therefore, $4 \pi \hat{C} N_{0} \mathbf{p}=\sum_{k \neq 0} \mathbf{E}_{k}$. Using the electric field of a dipole $\mathbf{E}_{k}=\left(3 \mathbf{r}_{k}\left(\mathbf{r}_{k} \mathbf{p}\right)-r_{k}^{2} \mathbf{p}\right) / r_{k}^{5}$, the interaction matrix can be determined as follows:

$$
\hat{C}=\frac{V_{c e l l}}{4 \pi} \sum_{k \neq 0} \frac{3 \mathbf{r}_{k} \otimes \mathbf{r}_{k}-r_{k}^{2}}{r_{k}^{5}},
$$

where $\mathbf{r}_{k} \otimes \mathbf{r}_{k}$ denotes a dyad (in coordinate notation $(\mathbf{a} \otimes \mathbf{b})_{i j}$ $\left.=a_{i} b_{j}\right), k$ varies from $-\infty$ to $\infty$. For the 1D array of the slits, $\mathbf{r}_{k}=k P \mathbf{e}_{x}$. Then the only important component of the interaction matrix $C_{x x}$ equals

$$
C_{x x}=\frac{V_{c e l l}}{2 \pi P^{3}} \sum_{k \neq 0}|k|^{-3}=\frac{P^{2} h}{\pi P^{3}} \sum_{k=1}^{\infty} k^{-3}=\frac{\zeta(3) h}{\pi P},
$$

where $\zeta(x)=\sum_{k=1}^{\infty} k^{-x}$ is the Riemann Zeta function.

Thus, the effective permittivity of the periodic array for the $x$-polarized incident field takes the form

$$
\varepsilon_{e f f}=1+\left((4 \pi \kappa)^{-1}-C_{x x}\right)^{-1} .
$$

Transmission spectra calculated for slabs of different periods $P$ with effective parameters given by Eq. (28) are shown in Fig. 4. As well as field enhancement, the spectrum has a plateau and a falling part. For larger frequencies, the metal becomes transparent and transmission tends to unity. Detailed analysis of the transmission spectra is given in Sec. IV.

\section{NUMERICAL MODELING OF SLIT ARRAYS}

The modeling is performed for gold films (Eq. (22)) of thickness $h=20 \mathrm{~nm}$. Different approaches can be used for calculations of transmission and enhancement factors. Simulation of transmission relies heavily on a size of the computational domain because the evanescent field spreads far away from the perforated metal film. In our transmittance calculations, the air buffer superposed with space squeezing is taken around 2 to $10 \mu \mathrm{m}$, the biggest size being used for extremely high enhancements generating strong 


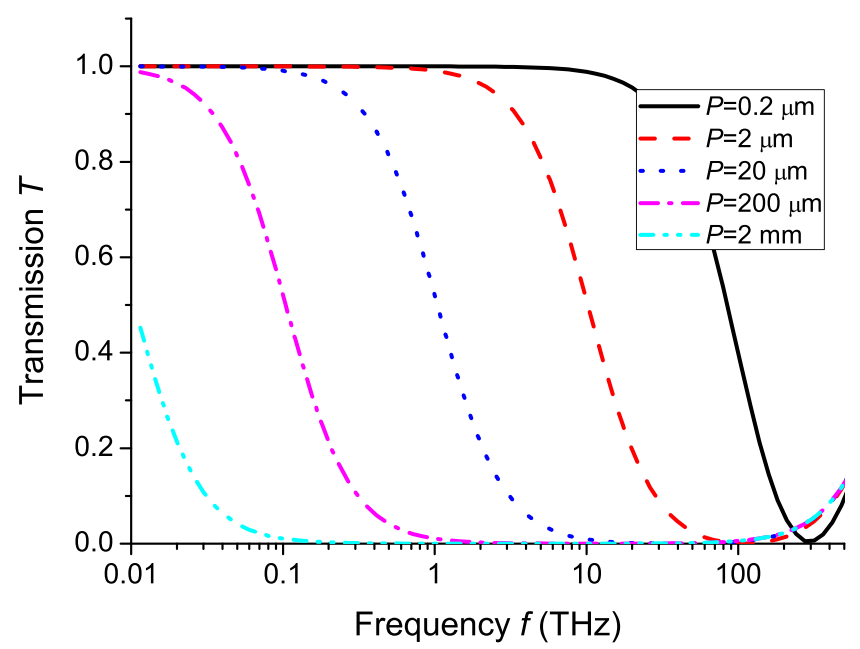

FIG. 4. Evolution of transmission spectra with period $P$ for thin metal films $(w=h=20 \mathrm{~nm})$. Spectra are calculated for the homogenized material with dielectric permittivity (28).

evanescent fields. Reliable calculations of $G$ are based on a fine enough discretization near the slit and the domain can be reduced further down to $1.5 \mu \mathrm{m}$ in favor of subnanometer grid step.

For both periodic and isolated slit structures, the buffer layers along the $z$-direction start right after the metal slab boundary and are squeezed with the $z /(1-z)$ function, one third of them is covered by the perfectly matched layers (PMLs). For isolated slits, the same buffers are used also along the $x$-direction where the squeezing includes metal plates. When calculating extra-narrow slits, e.g., $w=10 \mathrm{~nm}$, integration of the field along the air gap was substituted by a point value of the field in the center of the aperture (see Fig. 2(e) with uniform field in the narrow slit).

\section{A. 10-nm-wide slit in gratings of different periods}

In Fig. 5(a), we plot frequency dependence of the enhancement factor for a grating. Slit width $w=10 \mathrm{~nm}$ is kept constant while the period is changed from $20 \mathrm{~nm}$ to $100 \mu \mathrm{m}$ in a quasilogarithmic way. Thus, a broad variety of periods is examined and for each period from Fig. 5(a) there exists low-frequency range in which the simple static model works well. A constant enhancement $G=P / w$ is found with high precision for any ratio $P / w$ from 2 up to extreme $10^{4}$. The flat-enhancement behavior ends up with approaching the Rayleigh-Wood condition $\lambda=P$.

The transmission through a grating with varying period is shown in Fig. 5(b). At low frequencies, we see full transmission in agreement with static model. In the domain of flat enhancement, short $\mathrm{THz}$ and sub-THz pulses can be perfectly transmitted, experiencing the same enhancement in the near field of the gratings. The transmission has a stronger frequency dependence than the enhancement, meaning that it starts decreasing at frequencies approximately two orders of magnitude lower than the Rayleigh-Wood anomaly. For example, for the large enhancement $G=2000, f_{R-W}=15 \mathrm{THz}$ and roughly at $1 \mathrm{THz}$, the enhancement plateau breaks, while $T$ starts to decrease at even lower frequencies of about $0.1 \mathrm{THz}$.
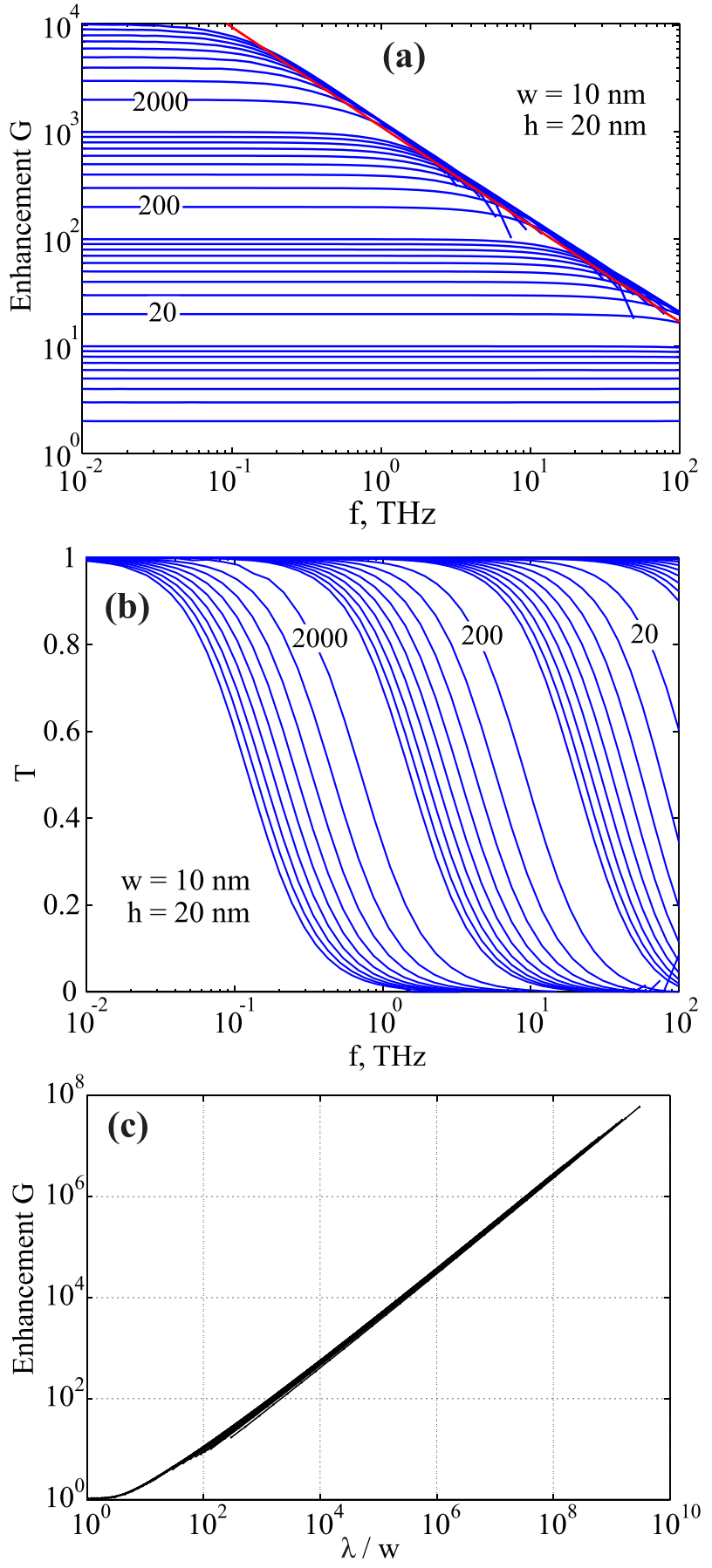

FIG. 5. (a) Field enhancement and (b) transmittance of 10-nm-wide slits cut in a 20 -nm-thick film versus frequency for different grating periods $P$ (FDFD simulation). The enhancements in the quasi-static limit are chosen to be $G=A \times 10^{B}$, where $A$ changes from 2 to 10 and $B$ from 0 to 3 (increment is 1 both for $A$ and $B$ ). The period of the grating is $P=w G$. (c) The dependence of the enhancement on the dimensionless parameter $\lambda / w$ for a single slit $(h=20 \mathrm{~nm})$. Each line is obtained by varying $\lambda$ at some slit width $w$, so that a total of 23 curves are plotted for a set of 23 slit widths (taking values between $10 \mathrm{~nm}$ and $2.9 \times 10^{3} \mathrm{~nm}$ ). For all the curves, the incident wavelength $\lambda$ is changed from $2.9 \times 10^{3} \mathrm{~nm}$ to $2.9 \times 10^{10} \mathrm{~nm}$ corresponding to the frequency range between $10^{-5}$ and $10^{2} \mathrm{THz}$.

For applications demanding a strong electric field (for nonlinear interactions), high transmission is inessential and can be sacrificed in favor of extended frequency range of large enhancement factor $G$. 
To compare the behavior of an isolated slit and a periodic grating, we plot the enhancement spectrum for a single slit of width $w=10 \mathrm{~nm}$ (red line in Fig. 5(a)). Since the increase of the period $P$ raises the enhancement factor $G$, the periodic structure cannot accumulate a larger charge and creates a stronger field than an isolated slit $(P \rightarrow \infty)$. This behavior is indeed seen in Fig. 5(a): the envelope formed by the enhancements in the periodic structures of different periods (blue line) almost coincides with the single slit curve (red line). Our analysis implies that for periodic structures, there are two regimes: $\lambda \gg P$ with total transmission and frequency-independent field enhancement $P / w$ (electrostatic approximation, when all slits operate cooperatively) and $\lambda \geq P$ (cooperative action of slits comes apart and each slit interacts with light rather as a separate scatterer).

The field in the single slit-capacitor is proportional to the potential difference at the slit edges and inversely proportional to the width $w$. This suggests us to operate with the dimensionless parameter $\lambda / w$ when we trace enhancement in the single slit for several slit widths in Fig. 5(c). Curve fitting for $\lambda / w>10^{4}$ reveals that the enhancement $P / w$ scales approximately as $(\lambda / w)^{0.93}$, i.e., very close to linear scaling. No limits in rising the field in the slit is seen: extremely high enhancements of $10^{8}$ are achieved at $\mathrm{MHz}$ frequencies. The strong fields can damage the nanoslits, and mechanical destruction can be considered as a limiting factor. For $\lambda / w<100$, the deviation from simple proportionality is more pronounced because slit dimensions become comparable to the incident wavelength and the capacitor approximation breaks.

Our simulations of 10 to $100 \mathrm{~nm}$ thick films with periodic or isolated slits $(P \rightarrow \infty)$ confirmed virtual independence of the enhancement on the slab thickness. However towards the optical range with faster field oscillations, a weak dependence of $G$ on the slab thickness might take place.

\section{B. Changing slit width in gratings with $P=20 \mu \mathrm{m}$}

Here, we investigate how the variation of the slit width affects the field enhancement. We fix the period of the gold grating $P=20 \mu \mathrm{m}$ and scan over different aperture widths. Such a value of period allows to cover the major part of $\mathrm{THz}$ range with very high enhancement. The widths are chosen such that in the electrostatic approximation, they provide enhancement factor of 50 to 1000 . The rule is quite simple: the narrower the slit, the bigger the field focusing effect. Fig. 6(a) demonstrates that electrostatic approximation with enhancement $G=P / w$ again works very well for a broad range of slit widths up to $400 \mathrm{~nm}$ wide opening. We see that flat enhancement zone size slightly changes from curve to curve, because the period is not changed and the RayleighWood anomaly is expected at the same frequency for different $w$. The threshold frequency between frequency independent and frequency dependent enhancement factors can be estimated from the intersection of the $P / w$ horizontal with the $(\lambda / w)^{0.93}$ line describing the single slit. This gives a very weak dependence of threshold frequency on $w$ while the period $P$ plays a key role. Note that in the case of $(\lambda / w)$ law
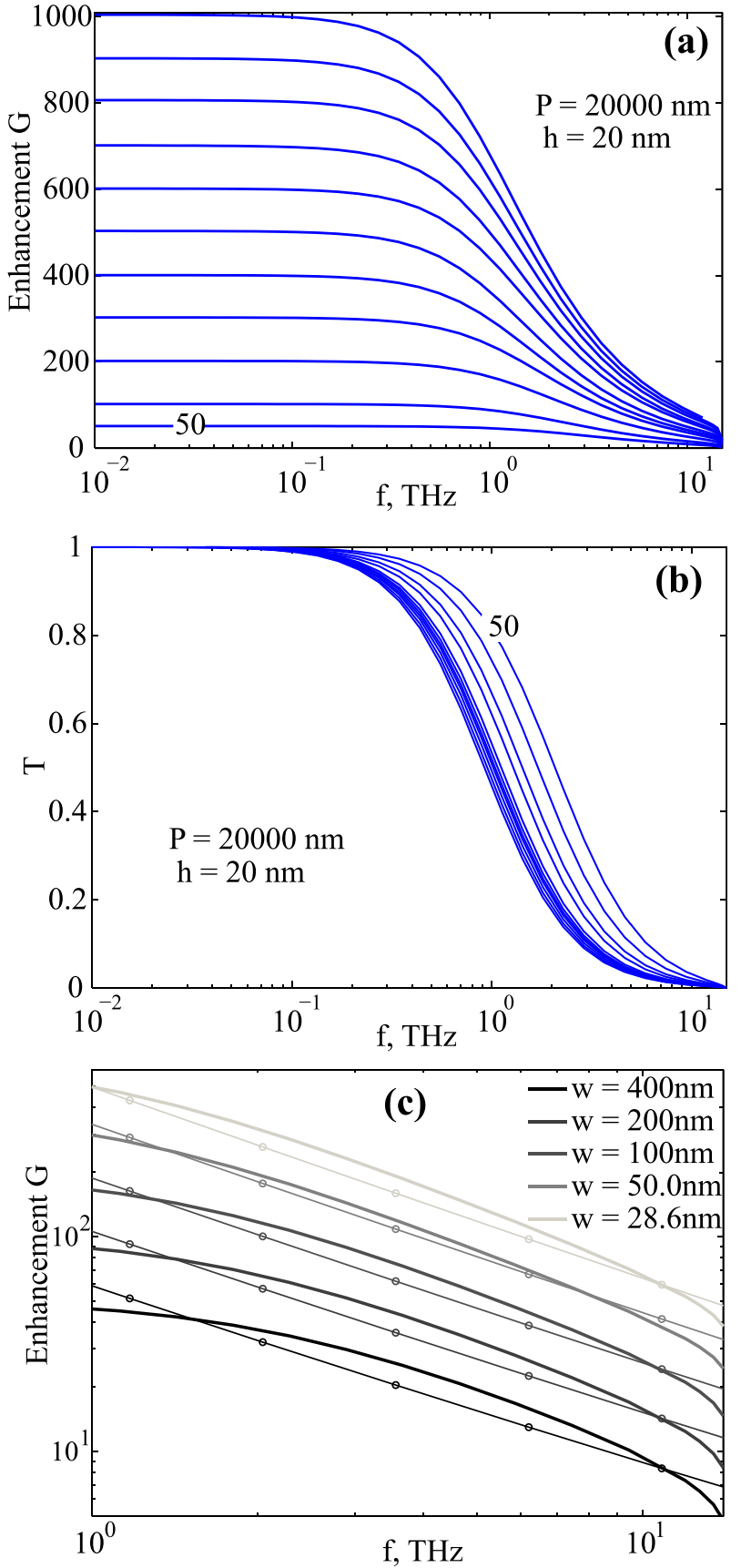

FIG. 6. (a) Enhancement $G$ and (b) transmittance $T$ in gratings with periodicity $P=20 \mu \mathrm{m}$ and varying slit width $w=P / G$ given by the following approximate set of numbers in $\mathrm{nm}: 400.0,200.0,100.0,66.7,50.0,40,33.3$, 28.6, 25.0, 22.2, and 20.0, which correspond to enhancements 50 and 100 to 1000 with the increment 100 within the plateau zone (FDFD simulation). (c) Detailization of several curves for the enhancement from (a) on a logarithmic scale. Lines with open circles correspond to the enhancement in isolated slits of the same widths as indicated in the legend.

for a single slit, threshold frequency would not depend on $w$. Thus, if higher enhancement is required without significant reduction of the flat zone, it is better implemented by reduction of the slit width. Total transmission observed in Figs. 5(b) and 6(b) at low frequencies agrees with previous experimental results ${ }^{19}$ where very long slits were analyzed and clear features of broadband performance were detected. This also resolves some of the speculations about connection between periodicity and transparency in the perforated films. ${ }^{31}$ 
In Fig. 6(c), we plot the transition region from the flat enhancement to the Rayleigh-Wood anomaly for the periodic slits and compare it to the enhancement in isolated slits of the same width. Although the curves display similar trends, different laws for the enhancement clearly show up: simple $1 / f$ dependence for the single slits and a somewhat more complicated behavior for periodic arrays.

\section{DISCUSSIONS AND CONCLUSIONS}

The results of Sec. IV for aperture gold films can be reproduced by employing the perfect electric conductor (PEC) model of metal instead of gold. The PEC model can emulate plasmonic behavior: surface bound $\operatorname{modes}^{8}$ or in an other terminology, spoof plasmons,${ }^{29}$ were shown to contribute to the EOT phenomena in perforated PEC films. Since metals at long wavelengths can be reasonably described as dispersionless perfect conductors, the condition for excitation of surface bound modes is the same in a wide range of frequencies, resulting in a flat, broadband field enhancement in the slits.

An incident $s$-polarized wave has a cutoff wavelength and its transmission is strongly suppressed at $\mathrm{THz}$ frequencies. However, additional cuts perpendicular to the slit can drastically change $s$-wave transmission ${ }^{30}$ indicating the relevance also for polarization other than $p$ if the metal film is perforated in a suitable manner.

In conclusion, we have discussed the huge non-resonant field enhancement of the electric field in the slit arrays, which dimensions are much smaller than the wavelength. For a single slit, the retardation effects affecting electrons lead to an increase of their effective collision frequency and, as a consequence, to an inverse frequency dependence of the field enhancement. The behavior of periodic arrays of slits is explained by non-resonant plasmonic oscillations. Here, we have obtained a simple law for the enhancement factor $G=P / w$ which depends only on the grating period $P$ and the slit width $w$. It is remarkable that enhancement is not influenced by changes of the metal thickness and frequency variations over a wide range. We have proposed a microscopic model to describe the physics behind the discussed phenomena. The model is able to predict both field enhancement and transmission starting from an equation of motion of the individual electrons and can be applied for the homogenization of metamaterials. ${ }^{32}$ The combination of controllable field concentration and total transmission paves the way for usage of metallic gratings for improvement of $\mathrm{THz}$ sources, detectors and sensors, as broadband polarizers and essentially for terahertz spectroscopy ${ }^{33,34}$ and modulation applications. ${ }^{35}$

\section{ACKNOWLEDGMENTS}

Support of the Danish Research Council for Technology and Production Sciences via the project $\mathrm{THz} \mathrm{COW}$ was acknowledged. Authors thank Dr. D. Shyroki for fruitful discussions.

\section{APPENDIX A: ELECTRIC FIELD OF A CHARGED STRIP}

A plane facet of a semi-infinite metal slab is a strip of width $h$ positioned at $x=x_{0}$ and infinite in the $y$-direction. Assume elementary electric charges $\eta d y_{0} d z_{0}$ are homogeneously distributed on the surface of the strip with surface charge density $\eta$. We assign each of such charges coordinates $\left(x_{0}, y_{0}, z_{0}\right)$ to calculate electric field $E_{x}$ of such strip, which is necessary for the estimation of enhancement factor $G$ (e.g., see Eq. (1)). An elementary charge produces electric field $d E_{x}=\eta d y_{0} d z_{0} \cos \varphi / r^{2}$ at point $P(x, y, z)$, where $r=$ $\sqrt{\left(x-x_{0}\right)^{2}+\left(y-y_{0}\right)^{2}+\left(z-z_{0}\right)^{2}}$ and $\varphi=\arccos ((x-$ $\left.x_{0}\right) / r$ ) is the angle to project the electric field vector onto the $x$ axis. Thus, the total electric field in the point $P$ is a superposition of all contributions from elementary charges

$$
E_{x}=\int_{-\infty}^{\infty} \int_{-h / 2}^{h / 2} \frac{\eta d y_{0} d z_{0}\left(x-x_{0}\right)}{r^{3}}
$$

Changing variable $y_{0}$ by $\rho$ as $y_{0}=y+\sqrt{\left(x-x_{0}\right)^{2}+\left(z-z_{0}\right)^{2}}$ $\tan \rho$ we get to

$$
E_{x}=\int_{-\pi / 2}^{\pi / 2} \int_{-h / 2}^{h / 2} \frac{\eta \cos \rho d \rho d z_{0}\left(x-x_{0}\right)}{\left(x-x_{0}\right)^{2}+\left(z-z_{0}\right)^{2}} .
$$

Calculating the integral over $\rho$ and changing the second variable as $z_{0}=z+\left(x-x_{0}\right) \tan \psi$ we arrive at

$$
E_{x}=2 \eta\left[\arctan \left(\frac{h / 2-z}{x-x_{0}}\right)+\arctan \left(\frac{h / 2+z}{x-x_{0}}\right)\right] .
$$

\section{APPENDIX B: FIELD ENHANCEMENT IN QUASISTATIC REGIME}

We consider a periodic array of slits with large periods $P \gg w, h$ in the quasistatic approximation $\left(\omega_{0} \gg \sqrt{\tilde{\gamma} \omega} \gg \omega\right)$. Then in Eq. (13), we neglect the sum over $j$ and terms $\omega^{2}$ and $\mathrm{i} \omega \tilde{\gamma}$ in the denominator, so that Eq. (13) is reduced to

$$
G_{c}=\left|1+\frac{2 \omega_{p}^{2}}{\pi \omega_{0}^{2}} \arctan \left(\frac{h}{w}\right)\right| .
$$

Frequency $\omega_{0}$, Eq. (9), is defined by the double integration of function $f$, Eq. (5). The difference of inverse tangent functions in Eq. (5) for $w \ll x, P$ can be approximated as

$$
\begin{aligned}
& \arctan \left(\frac{z_{+}}{x-j P+w / 2}\right)-\arctan \left(\frac{z_{+}}{x-j P-w / 2}\right) \\
& \approx \frac{d \arctan \left(z_{+} /(x-j P)\right)}{d(x-j P)} w .
\end{aligned}
$$

Then the sum of the terms involving $z_{+}$(similar $z_{-}$) and their integration over $x$ takes the form 


$$
\begin{aligned}
B & =\int_{-P+w / 2}^{-w / 2} \sum_{j=-\infty}^{\infty}\left[\arctan \left(\frac{z_{+}}{x-j P+w / 2}\right)-\arctan \left(\frac{z_{+}}{x-j P-w / 2}\right)\right] d x \\
& =w \sum_{j=-\infty}^{\infty} \int_{-P(1+j)+w / 2}^{-j P-w / 2} \frac{d \arctan \left(z_{+} / \xi\right)}{d \xi} d \xi=w \sum_{j=-\infty}^{\infty}\left[\arctan \left(\frac{z_{+}}{(1+j) P-w / 2}\right)-\arctan \left(\frac{z_{+}}{j P+w / 2}\right)\right] .
\end{aligned}
$$

The sum from $j=-\infty$ to -2 can be presented as

$$
\begin{aligned}
& \sum_{j=-\infty}^{-2}\left[\arctan \left(\frac{z_{+}}{(1+j) P}\right)-\arctan \left(\frac{z_{+}}{j P}\right)\right] \\
& =\sum_{j=-\infty}^{-1} \arctan \left(\frac{z_{+}}{j P}\right)-\sum_{j=-\infty}^{-2} \arctan \left(\frac{z_{+}}{j P}\right)=-\arctan \left(\frac{z_{+}}{P}\right) .
\end{aligned}
$$

In the similar way, it is possible to calculate the sum from $j=1$ to $\infty$, which results in $-\arctan \left(z_{+} / P\right)$ again. Writing down the two remain summands of the whole sum $B$ as

$$
\begin{gathered}
\sum_{j=-1}^{0}\left[\arctan \left(\frac{z_{+}}{(1+j) P}\right)-\arctan \left(\frac{z_{+}}{j P}\right)\right] \\
=2 \arctan \left(\frac{z_{+}}{P}\right)-2 \arctan \left(\frac{z_{+}}{w / 2}\right)
\end{gathered}
$$

we finally derive $B=-2 \arctan \left(2 z_{+} / w\right)$.

Performing in the similar form with the sum of the terms containing $z_{-}$and using $z_{ \pm}=h / 2 \pm z$, we eventually obtain

$$
\omega_{0}^{2}=\frac{\omega_{p}^{2} w}{\pi P h} \int_{-h / 2}^{h / 2}\left[\arctan \left(\frac{h / 2+z}{w / 2}\right)+\arctan \left(\frac{h / 2-z}{w / 2}\right)\right] d z .
$$

For small $2 z / w \ll 1$, we can apply the Taylor expansion arriving at the $z$-independent principle term in the integrand $2 \arctan (h / w)$. Thus

$$
\omega_{0}^{2}=\left(2 \omega_{p}^{2} w / \pi P\right) \arctan (h / w) .
$$

By substituting $\omega_{0}^{2}$ into Eq. (B1), we obtain $G_{c}=P / w$ (2). We would like to point out that condition $2 z / w \ll 1$ is applied here for performing analytical calculation only. Numerical evaluation of the original expressions for the $G$-(12) and $G_{c}$-factor (13) confirms that formula (2) is the perfect approximation of the enhancement factor for the periodic arrays of slits.

\footnotetext{
${ }^{1}$ R. W. Wood, Philos. Mag. 4, 396 (1902).

${ }^{2}$ R. W. Wood, Phys. Rev. 48, 928 (1935).

${ }^{3}$ H. A. Bethe, Phys. Rev. 66, 163 (1944).

${ }^{4}$ C. J. Bouwkamp, Rep. Prog. Phys. XVII, 35 (1954).

${ }^{5}$ G. W. Lehman, J. Math. Phys. 11, 1522 (1970).
}

${ }^{6}$ R. Harrington and D. Auckland, IEEE Trans. Antennas Propag. 28, 616 (1980).

${ }^{7}$ T. W. Ebbesen, H. J. Lezec, H. F. Chaemi, T. Thio, and H. F. Wolff, Nature (London) 391, 667 (1998).

${ }^{8}$ F. J. Garcia-Vidal, L. Martin-Moreno, T. W. Ebbesen, and L. Kuipers, Rev. Mod. Phys. 82, 729 (2010).

${ }^{9}$ J. A. Porto, F. J. Garcia-Vidal, and J. B. Pendry, Phys. Rev. Lett. 83, 2845 (1999).

${ }^{10}$ R. Gordon, Phys. Rev. B 73, 153405 (2006).

${ }^{11}$ H. E. Went, A. P. Hibbins, J. R. Sambles, C. R. Lawrence, and A. P. Crick, Appl. Phys. Lett. 77, 2789 (2000).

${ }^{12}$ J. R. Suckling, A. P. Hibbins, M. J. Lockyear, T. W. Preist, J. R. Sambles, and C. R. Lawrence, Phys. Rev. Lett. 92, 147401 (2004).

${ }^{13}$ B. Sturman, E. Podivilov, and M. Gorkunov, Phys. Rev. B 82, 115419 (2010).

${ }^{14}$ D. Wang, T. Yang, and K. B. Crozier, Opt. Express 18, 10388 (2010).

${ }^{15}$ D. J. Park, S. B. Choi, Y. H. Ahn, F. Rotermund, I. B. Sohn, C. Kang, M. S. Jeong, and D. S. Kim, Opt. Express 17, 12493 (2009).

${ }^{16}$ J. W. Lee, T. H. Park, P. Nordlander, and D. M. Mittleman, Opt. Express 17, 12660 (2009).

${ }^{17}$ J. H. Kang, D. S. Kim, and Q.-H. Park, Phys. Rev. Lett. 102, 093906 (2009).

${ }^{18}$ Q.-H. Park, Contemp. Phys. 50, 407 (2009).

${ }^{19}$ H. R. Park, Y. M. Park, H. S. Kim, J. S. Kyoung, M. A. Seo, D. J. Park, Y. H. Ahn, K. J. Ahn, and D. S. Kim, Appl. Phys. Lett. 96, 121106 (2010).

${ }^{20}$ M. A. Seo, H. R. Park, S. M. Koo, D. J. Park, J. H. Kang, O. K. Suwal, S. S. Choi, P. C. M. Planken, G. S. Park, N. K. Park, Q. H. Park, and D. S. Kim, Nature Photon. 3, 152-156 (2009).

${ }^{21}$ A. M. Ivinskaya, D. M. Shyroki, and A. V. Lavrinenko, IEEE Trans. Antennas Propag. 59, 4155 (2011).

${ }^{22}$ P. U. Jepsen, R. H. Jacobsen, and S. R. Keiding, J. Opt. Soc. Am. B 13, 2424 (1996)

${ }^{23}$ P. U. Jepsen, D. G. Cooke, and M. Koch, Laser Photonics Rev. 5, 124 (2011).

${ }^{24}$ A. Novitsky, M. Zalkovskij, R. Malureanu, and A. Lavrinenko, Opt. Commun. 284, 5495 (2011).

${ }^{25}$ A. D. Boardman, Electromagnetic Surface Modes (John Wiley and Sons, Chichester, 2003).

${ }^{26}$ S. Raza, G. Toscano, A.-P. Jauho, M. Wubs, and N. A. Mortensen, Phys. Rev. B 84, 121412 (2011).

${ }^{27}$ M. Walther, D. G. Cooke, C. Sherstan, M. Hajar, M. R. Freeman, and F. A. Hegmann, Phys. Rev. B 76, 125408 (2007).

${ }^{28}$ A. Ishimaru, S.-W. Lee, Y. Kuga, and V. Jandhyala, IEEE Trans. Antennas Propag. 51, 2550 (2003).

${ }^{29}$ J. B. Pendry, L. Martín-Moreno, and F. J. Garcia-Vidal, Science 305, 847 (2004).

${ }^{30}$ J. R. Suckling, J. R. Sambles, and C. R. Lawrence, Phys. Rev. Lett. 95, 187407 (2005).

${ }^{31}$ J. W. Lee, M. A. Seo, D. H. Kang, K. S. Khim, S. C. Jeoung, and D. S. Kim, Phys. Rev. Lett. 99, 137401 (2007).

${ }^{32}$ A. V. Novitsky, V. M. Galynsky, and S. V. Zhukovsky, Phys. Rev. B 86, 075138 (2012).

${ }^{33}$ B. Gelmont, R. Parthasarathy, T. Globus, A. Bykhovski, and N. Swami, IEEE Sens. J. 8, 791 (2008).

${ }^{34}$ M. Kutteruf, C. M. Brown, L. K. Iwaki, M. B. Campbell, T. M. Korter, and E. J. Heilweil, Chem. Phys. Lett. 375, 337 (2003).

${ }^{35}$ H. T. Chen, W. J. Padilla, M. J. Cich, A. K. Azad, R. D. Averitt, and A. J. Taylor, Nat. Photonics 3, 148 (2009). 